\title{
edmetic
}

Revista de Educación Mediática y TIC

\section{Liderazgo y e-liderazgo en las historias de vida de líderes \\ educativos a través del mundo}

\section{Leadership and e-leadership in the life histories of educational leaders throughout the world}

\author{
Mireia Tintoré ${ }^{1}$, Isabel Cantón Mayo ${ }^{2}$, Marta Quiroga Lobos ${ }^{3}$ e Isabel Parés ${ }^{4}$ \\ Fecha de recepción: 18/03/2019; Fecha de revisión: 26/04/2019; Fecha de aceptación: \\ 02/05/2019
}

\section{Cómo citar este artículo:}

Tintoré, M., Cantón Mayo, I., Quiroga Lobos, M., \& Parés, I. (2019). Liderazgo y e-liderazgo en las historias de vida de líderes educativos a través del mundo. EDMETIC, Revista de Educación Mediática y TIC, 8(2), 17-36. doi: https://doi.org/10.21071/edmetic.v8i2.11700

Autor de Correspondencia: $\underline{\text { mtintore@vic.es }}$

\section{Resumen:}

El liderazgo en los centros educativos ha de tener cada vez más en cuenta el papel de las nuevas tecnologías de la información y la comunicación, como medio para mejorar las organizaciones. En consecuencia, el objetivo de la presente investigación es analizar las historias de vida de 5 líderes educativos en 5 contextos diferentes: España, Guatemala, Chile, Kazajistán y Estados Unidos; y describir cómo las nuevas tecnologías se engarzan con sus prácticas de liderazgo. La metodología es cualitativa usando como instrumento entrevistas semiestructuradas recogidas entre 2012 y 2018 en los distintos entornos. Se ofrecen ejemplos de líderes, sus prácticas de liderazgo y, particularmente, el papel de los medios tecnológicos en su gestión del cambio. Los resultados revelan prácticas directivas similares y ajustadas a lo descrito previamente por la literatura académica, y muestran asimismo cómo liderazgo y tecnología se afectan mutuamente

Palabras clave: Liderazgo educativo; E-Liderazgo; Directores de centro; Historias de Vida.

\begin{abstract}
:
Leadership in educational centers must increasingly consider the role of advanced information technologies to improve organizations. Consequently, the objective of this research is to analyze the life histories of 5 educational leaders in 5 different contexts: Spain, Guatemala, Chile, Kazakhstan and the United States; and describe how new technologies are linked with their leadership practices. The methodology is qualitative using as instrument semi-structured interviews collected between 2012 and 2018 in different environments. Examples of leaders, their leadership practices and, mainly, the role of technological media in their change management are offered. The results reveal similar management practices and adjusted to what was previously described by the academic literature, and also show how leadership and technology affect each other.
\end{abstract}

Key Words: Educational Leadership; E-Leadership; Principals; Life Histories.

\footnotetext{
1 Universidad Internacional de Cataluña (España), mtintore@uic.es; $\underline{3691}$

2 Universidad de León (España), icanm@unileon.es; (1) https://orcid.org/0000-0002-9757-8233

3 Pontifica Universidad Católica de Valparaíso (Chile), marta.quiroga@pucv.cl; (D) https://orcid.org/0000-
} $\underline{0003-3630-384 X}$

4 Universidad Panamericana (México), isapares@yahoo.com; (1) https://orcid.org/0000-0001-7098-9908 


\section{INTRODUCCIÓN}

Las investigaciones sobre liderazgo educativo ocupan un espacio importante en los estudios sobre organización escolar. Desde los años finales del siglo XX, el término e-liderazgo ha ido ganando fuerza en la literatura sobre liderazgo, ampliándose recientemente el alcance de este concepto, desde posiciones que se ocupan de cómo los líderes usan las nuevas tecnologías cuando interactúan virtualmente hasta «considerar como las tecnologías avanzadas de la información y el liderazgo - en su sentido más amplio- se afectan mutuamente a lo largo del tiempo, espacio y culturası) (Avolio, Sosik, Kahai y Baker, 2014, p. 105).

Considerando la importancia de las nuevas tecnologías como medio para mejorar las organizaciones, y su relación con el liderazgo, el objeto de esta investigación es analizar cinco historias de vida de otros tantos líderes educativos, y poner de manifiesto sus prácticas de liderazgo y el papel que las tecnologías juegan en su función directiva y liderazgo.

Las historias de vida se han revelado como potentes instrumentos para conocer aspectos personales y sociales que no se abarcan desde los modelos cuantitativos. La elaboración de historias vitales ha permitido avanzar en el conocimiento de los líderes educativos eficaces, contribuyendo a mostrar que esos líderes comparten una serie de conocimientos, competencias y disposiciones (Leithwood, Lovis, Anderson y Wahldstrom, 2004), además de utilizar unas prácticas de liderazgo muy similares (Hallinger, 2009).

Enmarcado en este tipo de investigación narrativa, el presente estudio muestra y compara las historias de vida de cinco directivos escolares que ejercen su liderazgo en diferentes contextos. Las historias de vida muestran las trayectorias de los directivos escolares objeto de estudio, resumen sus prácticas de dirección, y describen cómo los líderes se apropian de la tecnología y cómo la tecnología impacta en su liderazgo (Avolio et al., 2014: 106).

Para lograr los objetivos y contribuir al desarrollo directivo, las historias de vida se elaboran teniendo en cuenta las experiencias e influencias de los cinco líderes educativos, sus principales retos y motivaciones, y sus principales decisiones profesionales. Asimismo, se analiza si las principales prácticas de 
liderazgo descritas por la literatura académica (Day, Sammons, Hopkins, Harris, Leithwood, Gu y Brown,2010; Leithwood, Day, Sammons, Harris y Hopkins,2006; Marzano, 2012) están presentes en las vidas de estos profesionales. Y se describe la forma en que cada líder utiliza las tecnologías que facilitan la expansión del conocimiento, para mejorar sus instituciones.

Diversos motivos justifican la realización de un estudio que ahonda en la figura y papel de los líderes escolares y su relación con las TIC: en primer lugar, por la complejidad creciente del rol del director (Dempster, Carter, Freakley y Parry, 2004), y de los contextos escolares en lo que se refiere a la tecnología (Esguerra y Contreras, 2016); además, por la decisiva influencia que el liderazgo tiene sobre la calidad de un centro educativo (Arias y Cantón, 2005) y por la relevancia que tiene el liderazgo educativo en la integración de las TIC en las aulas (Moureira, Hernández Rivero y Sosa Alonso, 2019; Fisher y Waller, 2013). Para finalizar, porque se necesitan con urgencia relatos de cómo los líderes transforman los procesos sociales de una manera efectiva y que responde al contexto (Harris y Jones, 2017; Shamir, Dayan-Horesh y Adler, 2005).

\section{MÉTODO Y DESARROLLO DE LA INVESTIGACIÓN}

El objetivo general de la investigación era conseguir una perspectiva general de la dirección y el liderazgo en diferentes contextos; conocer a las personas que ejercen la dirección, sus motivaciones, sus aspiraciones, sus realizaciones y el papel que las TIC juegan en su función directiva. Para ello se seleccionó el paradigma cualitativo, utilizando la metodología de análisis narrativo para elaborar historias de vida (Bamberg, 2010; Clandinin, 2013; Creswell y Poth, 2018; Flint, 2009; Fraser, 2004; Lieblich, Tuval y Zilver, 1999).

Las historias de vida resultan un buen instrumento cuando se investigan problemas del mundo real (Fraser, 2004); asimismo, la investigación narrativa es un modo democrático de indagar ya que otorga voz a los protagonistas de la investigación, convirtiéndolos en co-investigadores de sus propias vidas (Moriña, 2017).

De esta forma, se han elaborado relatos únicos de cinco líderes educativos, que al final se han convertido en un relato múltiple, que contempla «varios casos o historias de personas en torno a una misma realidad» (Pujadas, 2002, p. 29). Son historias de vida temáticas, centradas en el liderazgo y el e-liderazgo, y narradas en primera o tercera persona por cada 
investigadora. Las historias son monódicas (Moriña, 2017) pues sólo existe la voz del entrevistado, aunque algunas veces asoman levemente otros personajes.

Los instrumentos usados para la investigación han sido entrevistas semiestructuradas y la muestra ha sido intencional en cada contexto y en cada país. Las entrevistas han sido grabadas y se ha procedido a la depuración de datos antes de realizar el análisis de contenido (Bardin, 1996; Klipendorf, 1990).

Los líderes escogidos son directivos escolares exitosos que trabajan en España, Chile, Guatemala, Kazajistán y Estados Unidos. Los casos y países se han seleccionado a conveniencia de las cuatro investigadoras bien por proximidad geográfica, bien por tratarse de líderes con los que las investigadoras colaboran. Cada investigador realiza las entrevistas con el directivo correspondiente en base a un protocolo común, y los participantes firman un consentimiento informado accediendo a participar en la investigación. Para preservar el anonimato de los directivos, se crean los pseudónimos que se utilizan en estas páginas.

El proceso de creación de historias de vida se inicia con las entrevistas a directivos y directivas en las que narran su relato de vida o life story. Posteriormente, cada investigadora crea la historia (life history) correspondiente a cada relato, en colaboración y relación dialógica con el entrevistado intentando reducir la brecha entre la historia contada y la historia escrita, y permitiendo a los narradores revisar su relato.

Posteriormente, se procede al análisis de los datos organizándolos en temas y categorías. Para el propósito de esta investigación, nos hemos centrado en analizar dos temas: las prácticas y dimensiones de liderazgo, y el papel de las nuevas tecnologías en la dirección y en la gestión del cambio. El proceso finaliza con el análisis, comparación y síntesis de los resultados de las entrevistas en relación con estos dos temas, de cara a obtener las conclusiones del estudio.

\subsection{Marco teórico: liderazgo y e-liderazgo educativo}

El liderazgo educativo puede definirse como el proceso usado por los líderes para dar propósito a los esfuerzos de los miembros de una organización educativa, a la vez que les mueven a trabajar en colaboración en un ambiente de confianza y respeto (Green, 2013). 
Tras décadas de investigación, han podido establecerse una serie de premisas que caracterizan el liderazgo educativo efectivo: en primer lugar, se proponen modelos de liderazgo distribuido, transformacional, inclusivo y para el aprendizaje (Gumus et al., 2018; MacBeath y Dempster, 2009).

En segundo lugar, se considera que el liderazgo es un factor clave para la educación de calidad y la eficacia educativa. Se constata que, después de la labor del profesor en el aula, el liderazgo es el segundo factor en influencia sobre el aprendizaje (Day et al., 2010; Hallinger y Huber, 2012; Heck y Hallinger, 2009; Leithwood et al., 2006; Leithwood y Sun, 2009; Robinson et al., 2009).

Para conseguir un modelo de liderazgo que sea a la vez pedagógico, transformador, y distribuido, los líderes han de desarrollar una serie de prácticas identificadas en el informe para la NCSL por Leithwood y colaboradores (2006): establecer una dirección, desarrollar a las personas, rediseñar la organización y gestionar la enseñanza-aprendizaje. Posteriormente (Day et al., 2010), se incorporaron ocho dimensiones de liderazgo relacionadas con las cuatro practicas anteriores: definir valores y visión para aumentar las expectativas; establecer una dirección y generar confianza; remodelar las condiciones de enseñanza y aprendizaje; reestructurar la organización y rediseñar las funciones y responsabilidades del liderazgo; enriquecer el plan de estudios; mejorar la calidad de los docentes, incluyendo la planificación de la sucesión; mejorar la calidad de la enseñanza y el aprendizaje; construir colaboración interna y establecer relaciones sólidas fuera de la comunidad escolar.

Todas las prácticas y dimensiones han sido analizadas en esta investigación en relación con el contexto en el que se producen (Bush 2018; Gurr, 2015; Hallinger, 2018) pues la forma en que los líderes más efectivos aplican sus prácticas básicas de liderazgo «demuestran que son capaces de dar respuesta a los retos del contexto en el que trabajan, en lugar de ser dirigidos por élı (Leithwood et al., 2006, p.1).

Parte crucial del actual contexto educativo son las $\mathrm{TIC}$, hecho que explica la aparición del e-liderazgo en los años finales del siglo XX. La literatura referida a este nuevo tipo de liderazgo es menos numerosa y más reciente que la anterior (Avolio et al., 2001, 2003, 2007, 2014; Yee, 2000) pero está experimentando un gran crecimiento en los últimos años (Arnold y Sangrá, 2018; Dasgupta, 2011; Gilstrap y Hendershot, 2015; Moureira, Hernández Rivero 
y Sosa Alonso, 2019; Fisher y Waller, 2013). Se trata de un tema emergente dentro de los estudios de liderazgo educativo que ha sido definido por el grupo de Avolio como un «proceso de influencia social, integrado en contextos próximos y distantes, y mediado por las Tecnologías Avanzadas de la Información; que puede producir un cambio en las actitudes, los sentimientos, el pensamiento, el comportamiento y el rendimientor) (Avolio, Sosik, Kahai, y Baker, 2014). Con base en esta definición, el e-liderazgo ha de entenderse en dos sentidos: la intervención de las TIC en los procesos de liderazgo, y la influencia del liderazgo en la adopción de las TIC por parte de todos los miembros de la organización (Avolio et al., 2014).

Como señalan Esquerra y Contreras (2016), el e-liderazgo convive con las TIC, las utiliza y además contribuye a su aplicación y desarrollo. De esta forma las TIC se convierten en factores críticos a nivel individual (incluyendo a los propios líderes), grupal y organizacional.

En las páginas que siguen se presentan las prácticas de liderazgo y la respuesta al nuevo contexto tecnológico que se desprenden de las cinco historias de vida a directoras y directores en muy diferentes contextos.

\subsection{Cinco historias de vida: cinco líderes}

\section{Julio, un maestro rural (España)}

Julio afirma que se hizo maestro porque destacaba como estudiante, y sus maestros y familia lo animaron a continuar los estudios. Sus intereses estaban orientados a trabajar en la empresa minera de la zona, pero rápido entendió las oportunidades que le podrían brindar unos estudios universitarios, y su vocación se decantó hacia la docencia. Es de destacar que su madre era ama de casa y su padre trabajaba en la mina y en la zona no había muchas perspectivas profesionales en las que fijarse, la más cercana aparte de lo que tenía en casa eran los maestros, queridos y respetados en el pueblo. Además, en su provincia se podían estudiar otras carreras, pero eran más costosas, más largas y suponían un desembolso económico que muchas de las familias de las zonas rurales no podían afrontar, por lo que la elección primera se reforzó por la circunstancia contextual.

Hoy tiene 32 años de profesión como maestro y 17 años como director del centro. Sin embargo, matiza que «yo soy maestro que por determinadas razones desempeño labores de director, pero que tengo muy claro que por 
encima de todo soy maestroı. La iniciativa para ser director partió de un grupo de compañeros que le animan a presentar un proyecto de dirección. Se sometió el proyecto a la Administración educativa que lo seleccionó la primera vez y lo nombró para un periodo de 4 años que se ha prorrogado hasta la actualidad. Las innovaciones implantadas han seguido dos líneas principales, una es la mejora continua, basada en Planes de Mejora que le llevó a conseguir el premio al mejor servicio público de su Comunidad Autónoma en el año 2004. Otra línea es la aplicación de las Tecnologías de la Información y la Comunicación, al ámbito educativo. Todas las iniciativas que ha propuesto como director han contado con la participación de prácticamente el $100 \%$ de los compañeros/as. Desde hace un par de cursos está trabajando en implantar un Proyecto de autonomía en el Centro que permita cierta «maniobrabilidad» para diseñar un currículo adaptado a las características y necesidades de los alumnos. Y ha introducido innovaciones en el campo de la Educación para el Desarrollo que le ha valido un premio de la Fundación Vicente Ferrer. También ha recibido un reconocimiento autonómico y nacional por la calidad educativa del centro que dirige.

No se muestra fatigado ni cansado del cargo; al contrario, su función le sirve para mantener ese espíritu de hacer cada día algo nuevo y diferente sin caer en la rutina. El balance de este director es positivo, y la prueba es que la comunidad educativa le pide renovar en el cargo.

\section{Luis, relación constante con las empresas (Chile)}

Inicié mi carrera el año 1979 como profesor de Matemática y Física en un colegio técnico profesional. Después de cuatro años de trabajo fui invitado a colaborar con el Jefe de UTP, siendo este mi primer acercamiento a las tareas directivas, y lo que me motivó a realizar un curso de administración. Junto con el trabajo de apoyo al jefe de UTP, me familiaricé con la educación técnicoprofesional y con su importancia para los estudiantes de contextos más vulnerables que necesitan iniciar un trayecto laboral prontamente.

Después de algunos años, postulé a un trabajo de jornada completa en un establecimiento de educación secundaria municipal y cuando el director renunció, el alcalde me nombró director. Llevo 28 años en el cargo y, entre los diversos desafíos profesionales que esto me ha traído, sin duda el mayor fue el 
liderar la transformación desde liceo científico-humanista a técnicoprofesional.

Junto a esto, ha habido tres proyectos que han cambiado mi visión sobre el rol de director. El primero fue una pasantía en Francia, en la que participé en un curso sobre animación directiva. En él aprendí lo importante que es la motivación para el cambio y cómo los directivos pueden aumentar la motivación en sus equipos de profesores. El segundo fue una formación en Alemania que me permitió conocer y capacitarme en el modelo dual de formación, e implementar el modelo en el establecimiento. El tercero fue la participación, en un proyecto, en alianza con una universidad de la región, que me permitió profundizar en el uso de TIC en el establecimiento.

Mirando retrospectivamente la implementación del modelo dual, puedo afirmar que es la iniciativa que cambió al colegio y a la comunidad. El desafío de implementar un modelo dual se expresó en tres áreas: el currículo, los padres y apoderados, y la conformación de la red de la empresa. Actualmente me quedan dos años para jubilarme. Creo que el liceo puede vivir sin mí, me pregunto si yo podré vivir sin él.

\section{Hellen, un ejemplo de confianza y colaboración (Guatemala)}

A Hellen le fascina hablar y «contar historias» y utiliza estas habilidades con los alumnos y con el profesorado. Trata de ser honesta y de fomentar la honestidad entre sus profesores. Se manifiesta solidaria, responsable y empática. Su mayor compromiso, que intenta transmitir a los que de ella dependen, es «hacerlo todo en beneficio de los niños». Para ello, fomenta el diálogo constructivo con el profesorado y las familias, inculcando la atención y el respeto. También intenta cambiar las cosas de manera colaborativa: en la escuela, las decisiones se toman de forma consensuada; se llevan a la práctica entre todos a través de una planificación general y un plan concreto de acción; y son evaluadas por el profesorado y los padres. Suelen organizarse en comisiones y marcan sus objetivos en un Proyecto Educativo Institucional. Por ejemplo, uno de sus objetivos era «tener un espacio en el que el niño pudiera tener acceso a algo de tecnologían, y consiguieron formar parte de un proyecto que les proporcionó en 2014 un aula virtual para el establecimiento. Ahora se plantean nuevos objetivos y Hellen confía en 
poderlos alcanzar pues, como ella misma dice: usi no confío en mi trabajo y en lo que estoy haciendo, voy a dejar de hacer muchas cosas por miedo a no lograrlası. Esa confianza en sí misma y en los demás es el principal motor que impulsa las aspiraciones de Hellen.

\section{Joy, de la universidad a la escuela infantil (Estados Unidos)}

Hace tres años, representantes de su iglesia confiaron a la doctora Joy la tarea de buscar director para una escuela infantil. Así lo hizo, pero finalmente los responsables le pidieron que fuera ella misma quien dirigiera la escuela pues, pensaban que era la persona idónea. Aceptó con escaso entusiasmo pues use trataba de una posición que requería un cambio completolı. Sin embargo, se empleó a fondo para convertir la escuela en un lugar en el que colaborasen padres, profesores y alumnos y que pudiese competir con las instituciones de la zona. Al mismo tiempo que reestructuró los programas académicos de la institución, introdujo herramientas de tecnología educativa, que completaran los planes y programas de estudio y, a su vez, permitieran a la institución estar a la vanguardia.

Joy adoptó un currículum con nuevas estrategias de aprendizaje basadas en la investigación y en que profesores y alumnos participaran muy activamente, no sólo entre ellos, sino también con comunidades virtuales enfocadas a la educación en los primeros años de vida (early education). Para ello, se ocupó de la preparación del profesorado, y de la comunicación con los padres. A pesar de las resistencias iniciales, consiguió la unidad del profesorado y «los padres se maravillan de lo que aprenden los niños».

La colaboración virtual, en la que participaron los docentes se llama MyPeers y es definida como una plataforma de colaboración entre miembros de la comunidad educativa de educación inicial. Dentro de dicha plataforma se promueven tres estrategias fundamentales: lluvia de ideas, intercambio de estrategias educativas y se comparten recursos educativos de interés. Todo esto ocurre dentro del área específica de interés personal. En el caso de Joy, ella participó en el sub-grupo llamado Directors Corner, enfocado a las personas que ocupan puestos directivos. En general, el beneficio que tanto ella como los docentes encontraron, fue una gran cantidad de conexiones 
informales que sirvieron de inspiración para retar a una renovación académica y para profundizar en aspectos vitales que eran indispensables.

\section{Laia, modelo de capacidad emprendedora (Kazajistán)}

Nacida en una población mediterránea, Laia es directora y propietaria de dos escuelas de educación infantil en Kazajistán, donde lleva viviendo unos 17 años. Compagina su vida en el país con estancias en su lugar de origen, desde donde sigue en contacto con sus escuelas a través de las nuevas tecnologías, lo que le permite dirigir a distancia. El currículum de sus dos escuelas destaca por seguir el esquema de las escuelas locales incorporando el inglés y aspectos del currículum internacional, que son propios de las escuelas de élite del país. No es un mero cambio de currículum. También se trata de cambiar la mentalidad de los padres y de las maestras. Las maestras siguen el currículum pero cuesta que lo entiendan y hay que justificarlo, entusiasmarlas, darles explicaciones y enseñarles a tener iniciativa y a disentir. Algunas se van.

Cuando terminan el parvulario, los alumnos se trasladan a escuelas internacionales o escuelas públicas, donde superan sin dificultad el examen de entrada. Laia lamenta que los estudiantes que no van a centros internacionales pierden mucho de lo aprendido en estos primeros años. Ahora, su nuevo reto es ampliar con una escuela de primaria y continuar el trayecto emprendido por lo menos hasta la secundaria.

\section{RESULTADOS Y DISCUSIÓN}

La muestra del estudio (ver Tabla 1) es variada, se compone de dos hombres y tres mujeres que trabajan en cinco países diferentes pertenecientes a tres continentes distintos. Los directores y directoras trabajan en escuelas públicas (3) y privadas (2); tres escuelas son urbanas y dos de ámbito rural; además las escuelas incluyen todos los niveles educativos desde educación infantil hasta la universidad por lo que puede decirse que los participantes abarcan un amplio espectro de escuelas y contextos. 
Tabla 1. Descripción demográfica de los participantes en el estudio

\begin{tabular}{|c|c|c|c|c|}
\hline $\mathrm{N}^{\circ}$ & Director-a & Sexo & Tipo de escuela & País \\
\hline 1 & Julio & $M$ & Primaria. Rural. Pública. & España \\
\hline 2 & Luis & M & $\begin{array}{l}\text { Liceo técnico-profesional. Urbana. } \\
\text { Pública }\end{array}$ & Chile \\
\hline 3 & Hellen & $\mathrm{F}$ & $\begin{array}{l}\text { Escuela infantil y primaria. Rural. } \\
\text { Pública }\end{array}$ & Guatemala \\
\hline 4 & Joy & $\mathrm{F}$ & Escuela infantil. Urbana. Privada & $\begin{array}{l}\text { Estados } \\
\text { Unidos }\end{array}$ \\
\hline 5 & Laia & $\mathrm{F}$ & Escuela infantil. Urbana. Privada & Kazajistán \\
\hline
\end{tabular}

El contexto en el que se desenvuelven estos directivos presenta diferentes retos y formas de afrontarlos, tal como puede observarse en la Tabla 2.

Tabla 2. Principales cambios emprendidos por los participantes en la investigación

\begin{tabular}{|c|c|c|c|c|}
\hline$n$ & Nombre & Cambios/Retos & $\begin{array}{l}\text { Prácticas } \\
\text { específicas }\end{array}$ & Estrategia \\
\hline 1 & Julio & $\begin{array}{l}\text { Mejorar con base en planes de } \\
\text { mejora y TIC } \\
\text { Luchar por elevar la calidad } \\
\text { educativa de las zonas rurales } \\
\text { Compatibilizar la docencia, la } \\
\text { dirección y las tareas } \\
\text { administrativas es su principal } \\
\text { dificultad }\end{array}$ & $\begin{array}{l}\text { Objetivo claro: } \\
\text { implantar } \\
\text { innovaciones } \\
\text { Crear } \\
\text { colaboración: } \\
\text { evolución de } \\
\text { dirección } \\
\text { personalista a } \\
\text { colegiada } \\
\text { Innovaciones } \\
\text { docentes y } \\
\text { premios }\end{array}$ & $\begin{array}{l}\text { Crear grupos } \\
\text { para distribuir } \\
\text { el liderazgo } \\
\text { Colaboración } \\
\text { total de los } \\
\text { compañeros }\end{array}$ \\
\hline 2 & Luis & $\begin{array}{l}\text { Transformar el centro de liceo } \\
\text { científico-humanista, a escuela } \\
\text { técnico profesional con modelo } \\
\text { dual y gran importancia a las } \\
\text { TIC } \\
\text { Reforzar las relaciones con las } \\
\text { empresas para tener } \\
\text { estudiantes que se formen a la } \\
\text { vez que realizan prácticas }\end{array}$ & $\begin{array}{l}\text { Objetivo claro: } \\
\text { transformar a } \\
\text { centro dual } \\
\text { Fomento de } \\
\text { relaciones con las } \\
\text { empresas y con } \\
\text { los padres } \\
\text { Formación del } \\
\text { profesorado en la } \\
\text { universidad y en } \\
\text { las empresas de } \\
\text { prácticas } \\
\text { Cambios } \\
\text { curriculares }\end{array}$ & $\begin{array}{l}\text { Adaptación } \\
\text { del currículum } \\
\text { Conversacione } \\
\text { s y reuniones } \\
\text { con las } \\
\text { empresas y } \\
\text { con los padres }\end{array}$ \\
\hline 3 & Hellen & $\begin{array}{l}\text { Cambiar por completo } \\
\text { Conseguir más aulas y recursos } \\
\text { tecnológicos } \\
\text { Introducir nuevas tecnologías en } \\
\text { las aulas }\end{array}$ & $\begin{array}{l}\text { Objetivo claro: } \\
\text { cambiar la } \\
\text { imagen del } \\
\text { centro } \\
\text { Mejora su }\end{array}$ & $\begin{array}{l}\text { Busca ayuda } \\
\text { en ONGs y } \\
\text { pide } \\
\text { subvenciones } \\
\text { Diálogo con el }\end{array}$ \\
\hline
\end{tabular}


4 Joy

5 Laia
Ser comparable a las escuelas de la zona, lo que requiere un cambio completo.

Convertir la escuela en un ámbito de colaboración de toda la comunidad educativa aprovechando las TIC Implantar innovaciones curriculares que mejoren el aprendizaje y sean aceptadas por las familias

Desarrollar un proyecto nuevo y diferente en Kazajistán que puede seguir a distancia a través de las TIC

Reforzar el pensamiento crítico del profesorado Implantar innovaciones curriculares internacionales, junto a lo propio del país capacitación y la del profesorado

Fomenta el diálogo y la colaboración Objetivo claro: hacer competitiva la escuela Atención y apoyo intelectual y técnico al profesorado Relaciones productivas con las familias Cambios en el currículum

Objetivo claro: educación de calidad Atención y apoyo intelectual y técnico al profesorado Altas expectativas profesorado y

las familias Trabajo por medio de comisiones Compartir el conocimiento de la dirección con el profesorado Implicar a las familias en el currículum Firmeza Normas que permitan actuar con seguridad Formación continua del profesorado en la escuela Reuniones para desarrollar conocimiento y pensamiento crítico

En la Tabla 2 se observan los retos que deben afrontar cada director y directora, muchos de ellos relacionados con las TIC. También se incluyen las prácticas de liderazgo que ponen en práctica para lograr la transformación. Los retos son muy diferentes pues van desde la creación (Laia) y la remodelación total de las escuelas (Luis, Joy y Hellen), a la introducción de cambios concretos (Julio). Para incorporar innovaciones curriculares que mejoren el aprendizaje, los directores implementan estrategias de colaboración entre colegas, ofrecen apoyo directo y brindan oportunidades para la capacitación continua, utilizando para ello todos los medios tecnológicos a su disposición (Julio, Luis, Joy, Laia), o luchando para poder obtenerlos (Hellen).

Analizados estos datos a la luz de las prácticas y dimensiones de liderazgo, podemos observar, como muestra la columna 4 (Tabla 2) que todos los participantes en el estudio desarrollan las prácticas y dimensiones de liderazgo señaladas en los informes internacionales citados (Leithwood et al., 2006; Day et al., 2010): tienen una visión (un reto) y establecen una dirección 
para alcanzarlo. Para ello, se ocupan de su formación y del desarrollo de las personas a su cargo, y rediseñan la organización centrándose sobre todo en la gestión de la enseñanza-aprendizaje (enriquecimiento del plan de estudios, mejora de las infraestructuras y tecnología, mejora de las prácticas escolares), y en los aspectos de convivencia (generan colaboración dentro de la escuela y con las familias; crean relaciones sólidas con la comunidad externa). En este proceso, las TIC juegan un papel relevante, lo que permite reconocer la existencia de liderazgo y e-liderazgo.

La innovación, creatividad y puesta al día es algo que destaca en las trayectorias laborales de estos directivos, por ejemplo, en el director de escuela rural española que dedica sus esfuerzos innovadores a la aplicación de las TIC en su campo educativo; o en el director chileno, Luis, cuya innovación se ve estimulada por procesos formales de desarrollo profesional y desarrollo de las TIC en alianza con la universidad.

En 2001, Marion y Uhl-Bien señalaron que, para crear innovación, los líderes debían centrarse en desarrollar las condiciones necesarias para provocar el cambio. Una de esas condiciones son los medios tecnológicos, y la investigación muestra cómo - en diferentes grados y formas - todos y cada uno de los participantes en el estudio aprovechan las TIC para la gestión del cambio, para mejorar su acción directiva o para mejorar la calidad de la educación.

Los directivos han enfrentado el desarrollo profesional de los profesores a través del aprendizaje entre pares, lidiando con condiciones estructurales y físicas adversas de las escuelas (Little, 1990; Rosenholtz, 1989); han gestionado las capacidades de los profesores para lidiar con posiciones divergentes (Grossman, Wineburg y Woolworth, 2001) y también con la falta de experiencia y formación para la colaboración (Day, 2005). Los directivos han centrado la colaboración entre docentes como una herramienta de mejora de los aprendizajes de los alumnos, lo que según Lavié Martínez (2004), aumenta la valoración de la interacción con colegas. Culturas colaborativas tienen más oportunidades de mejorar los centros escolares (Donmoyer, Yennie-Donmoyer y Galloway, 2012; McLaughlin y Talbert, 2006; Sebastian y Allensworth, 2012; Vescio, Ross y Adams, 2008) y todos esos casos, las TIC han facilitado dicha 
colaboración para mejorar la calidad educativa, tal como muestra el caso de Joy.

A partir de estos cinco casos, se concluye que los directores se caracterizan por visionar futuros deseables que alcanzan liderando la innovación e incorporando las TIC para solucionar problemas de la organización y mejorar la enseñanza-aprendizaje. Los desafíos con los que se encuentran los directores están asociados a muy diversos aspectos, y las estrategias de solución son colaborativas, con mayor o menor peso del director o directora, según sus características personales y las características del contexto.

Nuestro estudio coincide con el de otros autores (i.e. Johnson, Moller, Jacobson y Wong, 2008), que han comparado prácticas de liderazgo de éxito en directivos de diversos países. Nuestra investigación indica también que las prácticas de liderazgo desarrollan espacios de colaboración y confianza en las escuelas (Gurr, 2015; Jacobson y Ylimakli, 2011; Johnson et al., 2008) favorecidos por el uso de las TIC, y son sensibles al contexto nacional, cultural e institucional.

El equipo investigador es consciente de las limitaciones de este estudio, particularmente por el hecho de que son únicamente los líderes quienes describen su historia de vida, sin que los datos se hayan triangulado con la observación de sus prácticas ni con opiniones de otros miembros de la comunidad. No obstante, los directivos participantes han sido elegidos por las investigadoras en base a su reputación como líderes, y la suma de las características de todas las historias, así como las grandes coincidencias halladas entre ellas, puede fortalecer la validez interna de la investigación (Flint, 2009) y mejorar el conocimiento sobre el liderazgo en entornos educativos.

\section{4, CONCLUSIONES}

Aunque existen diferencias notables en la forma en que los participantes en el estudio se relacionan con el contexto y en sus trayectorias profesionales, las principales características de los directores participantes en este estudio son muy similares. Los directivos desarrollan de forma natural las prácticas de liderazgo definidas por la comunidad científica internacional y los participantes usan las mismas prácticas, apoyándose para ello en las nuevas 
tecnologías de la información que facilitan el trabajo y la colaboración. Los directivos se plantean el uso de las tecnologías como medio para mejorar la calidad educativa en sus instituciones, las utilizan para construir comunidad educativa, para favorecer la dirección y liderazgo y para mejorar la gestión del conocimiento.

En un proceso bi-direccional, los líderes de este estudio usan las TIC para dirigir a distancia, para crear innovación, para renovar las estructuras de sus escuelas o para formar mejor a los alumnos proporcionándoles los medios tecnológicos que necesitan. Al mismo tiempo, los directivos son afectados por las TIC pues éstas actúan como «des-intermediadoras», permitiendo una mejor distribución del liderazgo y una mejora de la comunicación entre los diferentes grupos y estamentos (Avolio et al., 2014). De esta forma, TIC y liderazgo interactúan uno con otro afectando a los individuos, los grupos y toda la comunidad educativa (Avolio, Kahai y Dodge, 2000; Dasgupta, 2011; Gilstrap y Hendershot, 2015; Marion y Uhl-Bien, 2001). Esta investigación ha podido observar lo ya enunciado por Avolio y colaboradores en su revisión de 2014: que cuando los líderes- como en este caso - se apropian de la tecnología, ésta impacta también en el liderazgo.

Los resultados parecen indicar que el poder que tienen estos líderes no está relacionado con su posición o estatus, sino con su carrera profesional y su vida. Aunque tienen antecedentes culturales y niveles de estudio muy diferentes, los directores y directoras manifiestan un gran interés en el aprendizaje y en mantenerse siempre al día; centrándose en la estructuración de las escuelas como comunidades profesionales de aprendizaje. Asimismo, se puede afirmar que los líderes han sido capaces de salir de su zona de confort en las aulas o en otras ocupaciones, capacitándose y convirtiéndose en centro de una red de influencia y de aprendizaje en la que las tecnologías juegan un papel muy importante. Desde su posición, cada líder trata de desplegar competencias en las personas de su organización, y promover la mejora social en sus comunidades a través del desarrollo educativo.

Ahondando en el papel de los medios tecnológicos en el ejercicio de la dirección, las coincidencias encontradas entre estos líderes en contextos tan diferentes podrían facilitar una formación virtual conjunta en la que se reforzase el e-liderazgo. Un segundo paso podría ser «aprender a liderar a 
través de medios tecnológicos (...) tal como se esperaría que lo hicieran en forma no virtualı (Esguerra y Contreras, 2016: 264). Y finalmente, también podría crearse una red de líderes, de forma que pudiesen compartir retos, problemas, experiencias y buenas prácticas.

\section{REFERENCIAS}

ARIAS GAGO, A. R., y CANTÓN MAYO, I. (2005). El liderazgo y la dirección de centros educativos. Barcelona: Davinci Continental.

ARNOLD, D., y SANGRÁ, A. (2018). Dawn or dusk of the 5th age of research in educational technology? A literature review on (e-)leadership for technology-enhanced learning in higher education (2013-2017). International Journal of Educational Technology in Higher Education, 15, 1-29. doi: https://doi.org/10.1 186/s41239-018-0104-3

AVOLIO, B.J. (2007). Promoting more integrative strategies for leadership theory building. American Psychology, 62, 25-33.

AVOLIO, B. J., y KAHAI, S. S. (2003). Adding the E to e-leadership: How it may impact your leadership. Organizational Dynamics, 31, 325-338. doi: https://doi.org/10.1016/s0090-2616(02)00133-x

AVOLIO, B. J., KAHAI, S., y DODGE, G. E. (2001). E-leadership: Implications for theory, research, and practice. The Leadership Quarterly, 11 (4), 615-668. doi: https://doi.org/10.1016/s1048-9843(00)00062-x

AVOLIO, B. J., SOSIK, J. J., KAHAI, S. S., y BAKER, B. (2014). E-leadership: Reexamining transformations in leadership source and transmission. The Leadership Quarterly, 25, 105-131. doi: https://doi.org/10.1016/j.leaqua.2013.11.003

BAMBERG, M. (2010). Narrative Analysis. In: H. Cooper (ed.). APA Handbook of research methods in psychology. Washington D.C.: APA Press.

BARDIN, L. (1996). Análisis de contenido. $2^{a}$ edición. Madrid: Akal

BUSH, T. (2018). Research on Educational Leadership and Management: Broadening the Base. Educational Management Administration \& Leadership, 46 (3), 359-361. doi: https://doi.org/10.1177/1741143218758555

CLANDININ, D. J. (2013). Engaging in narrative inquiry. Walnut Creek, CA: Left Coast Press.

CRESWELL, J.W., y POTH, C.N. (2018). Qualitative Inquiry and Research Design: Choosing Among Five Approaches. Thousand Oaks, CA: Sage. 
DASGUPTA, P. (2011). Literature review: e-Leadership. Emerging Leadership Journeys, 4(1), 1-36.

DAY, C. (2005). Formar docentes: cómo, cuándo y en qué condiciones aprende el profesorado. Madrid: Narcea.

DAY, C., SAMMONS, P., HOPKINS, HARRIS, A., LEITHWOOD, K., GU, y BROWN. (2010). Ten strong claims about successful school leadership. UK: NCLSCS.

DEMPSTER, N., CARTER, L., FREAKLEY, M., y PARRY, L. (2004) Contextual influences on school leaders in Australia: some data from a recent study of principals' ethical decision-making. School Leadership \& Management: Formerly School Organization, 24(2), 163-174

DONMOYER, R., YENNIE-DONMOYER, J., y GALLOWAY, F. (2012). The search for connections across principal preparation, principal performance, and student achievement in an exemplary principal preparation program. Journal of Research on Leadership Education, 7(1), 5-43.

ESGUERRA, G.A., y CONTRERAS, F. (2016). Liderazgo electrónico, un reto ineludible para las organizaciones de hoy. Estudios Gerenciales, 32, 262268.

FISHER, D.M., y WALLER, L.R. (2013). The 21st Century Principal: A Study of Technology Leadership and Technology Integration in Texas K-12. The global e-learning journal, 2(4),

FLINT, L.J. (2009). Using life story research in gifted education. Gifted Children, $3(2)$, article 4, 5-12.

FRASER, H. (2004). Doing narrative research: Analysing personal stories line by line. Qualitative Social Work, 3, 179-198.

GILSTRAP, C., y HENDERSHOT, B. (2015). E-leaders and uncerTICnty management: A computer-supported qualitative investigation. Qualitative Research Reports in Communication, 16(1), 86-96. doi: https://doi.org/10.1080/17459435.2015.1086424

GROSSMAN, P., WINEBURG, S., y WOOLWORTH, S. (2001). Toward a theory of teacher community. Teachers College Record, 103(6), 942-1012.

GREEN, R.L. (2013). Practicing the art of leadership: A problem-base guide to learning in schools. Upper Saddle River, N.J.: Pearson Education, Inc.

GUMUS, S., BELLIBAS, M. S., ESEN, M., y GUMUS, E. (2018). A systematic review of studies on leadership models in educational research from 1980 to 2014. 
Educational Management Administration \& Leadership, 46(1), 25-48. doi: https://doi.org/10.1177/1741143216659296

GURR, D. (2015). A model of Successful School Leadership from the International Successful School Principalship Project. Societies, 5, 136-150. doi: https://doi.org/10.3390/soc5010136

HALLINGER, P. (2009). Leadership for 21 st Century Schools: From Instructional Leadership to Leadership for Learning. Hong Kong: The Hong Kong Institute of Education.

HALLINGER, P., y WANG, W.C. (2015). Assessing Instructional Leadership with the Principal Instructional Management Rating Scale. Switzerland: Springer. doi: https://doi.org/10.1007/978-3-319-15533-3

HALLINGER, P. (2018). Bringing context out of the shadows of leadership. Educational Management Administration \& Leadership, 46(1), 5-24. doi: https://doi.org/10.1177/1741143216670652

HARRIS, A., y JONES, M. (2017). Leading in context: Putting international comparisons into perspective. School Leadership \& Management, 37(5), 431-433. doi: https://doi.org/10.1080/13632434.2017.1368864

JACOBSON S.L., y YLIMAKI R.M. (2011). Comparative Perspectives: An Overview of Seven Educational Contexts. En: R. Ylimaki, y S. Jacobson (eds). US and Cross-National Policies, Practices, and Preparation. Studies in Educational Leadership, 12. Dordrecht: Springer.

JOHNSON, L., MOLLER, J., JACOBSON, S., y WONG, K.C. (2008). Cross-national comparisons in the International Successful School Principalship (ISSPP): the United States, Norway and China. Scandinavian Journal of Educational Research, 52(4), 407-422.

KRIPPENDORFF, K. (1990). Metodología de análisis de contenido. Teoría y Práctica. Paidós Comunicación.

LAVIÉ MARTíNEZ, J. M. (2004). Individualismo y privacidad en la cultura docente: revisando los argumentos. Revista Española de Pedagogía, 229, 439-454.

LEITHWOOD, K., DAY, C., SAMMONS, P., HARRIS, A., Y HOPKINS, D. (2006). Seven strong claims about successful school leadership. UK: NCSL.

LEITHWOOD, K., LOUIS, K.S., ANDERSON, S., y WAHLSTROM, K. (2004). Review of research: How leadership influences student learning. University of Minnesota: The Wallace Foundation. 
LEITHWOOD, K. y SUN, J-P, 2009, Transformational school leadership effects on schools, teachers, and students. (1-22) In W. K. Hoy y M. DiPaola (Eds). Studies in School Improvement. Charlotte, NC: Information Age Publishers.

LIEBLICH, A., TUVAL-MASHIAK, E., y ZILVER, T. (1999). Narrative Research. Reading, analysis, and interpretation. Thousand Oaks: SAGE.

LITTLE, J. (1990). The persistence of privacy: autonomy and initiative in teacher's professional relations. Teachers College Record, 91 (4), 509-535.

MACBEATH, J., y DEMPSTER, N. (2009) [Eds] Connecting Leadership and Learning, London, Routledge.

MARION, R., y UHL-BIEN, M. (2001). Leadership in complex organizations. The Leadership Quarterly, 12, 389-418. doi: https://doi.org/10.1016/s10489843(01)00092-3

MCLAUGHLIN, M., y TALBERT, J. (2006). Building School-Based Teacher Learning Communities. Professional strategies to improve student achievement. Nueva York: Teacher's College Press.

MARZANO, R. (2012). Marzano School Leadership Evaluation Model. Fl: Marzano Center.

MOREIRA, M. A., RIVERO, V. M. H., y ALONSO, J. J. S. (2019). Leadership and school integration of ICT. Teachers perceptions in Spain. Education and Information Technologies, 24(1), 549-565.

MORIÑA, A. (2017). Investigar con historias de vida. Metodología biográficonarrativa. Madrid: Narcea.

MOYLE, K. (2005). Leadership and Learning with information and communication technologies. Canberra ACT: Teaching Australia.

PUJADAS, J.J. (2002). El método biográfico: el uso de las historias de vida en ciencias sociales. Madrid: Centro de investigaciones sociológicas.

ROBINSON, V., LLOYD, C., y ROWE, K. (2008). The impact of leadership on student outcomes: An analysis of the differential effects of leadership types. Educational Administration Quarterly, 44(5), 635-674.

ROSENHOLTZ, S. (1989). Teachers Workplace: The Social Organization of Schools. White Planis, NY: Longman.

SEBASTIAN, J., y ALLENSWORTH, E. (2012). The influence of principal leadership on classroom instruction and student learning: A study of mediated 
pathways to learning. Educational Administration Quarterly, 48(4), 626663. doi: https://doi.org/10.1177/0013161X11436273

SHAMIR, B., DAYAN-HORESH, H., y ADLER, D. (2005). Leading by biography: Towards a life-story approach to the study of leadership. Leadership, 1 (1), 13-29. doi: https://doi.org/10.1177/1742715005049348

VESCIO, V., ROSS, D., y ADAMS, A. (2008). A review of research on the impact of professional learning communities on teaching practice and student learning. Teaching and Teacher Education, 24(1), 80-91.

YEE, D. L. (2000). Images of School Principals' Information and Communications Technology Leadership. Journal of Information Technology for Teacher Education, 9(3), 287-302. 